DOI https://doi.org/10.30525/978-9934-588-47-1.20

\title{
УПРАВЛЕНЧЕСКИЕ ЗАДАЧИ ОБЕСПЕЧЕНИЯ ЭФФЕКТИВНОГО ФУНКЦИОНИРОВАНИЯ И РАЗВИТИЯ ПРОИЗВОДСТВЕННОЙ ОРГАНИЗАЦИИ
}

\author{
Савенко В. И., Полосенко А. В.
}

\section{ВВЕДЕНИЕ}

В современных условиях эффективное развитие систем управления - одно из важнейших направлений повышения экономического потенциала строительных организаций. Опыт показывает, что организации, возглавляемые высококвалифицированными, опытными руководителями, в совершенстве владеющими современными знаниями и техникой, как правило, успешные.

В системе управления реализуется процесс взаимодействия объекта управления и управляющей подсистемы. Управляемость системы - одно из основных свойств, характеризующих состояние системы и ее организованность.

Вырабатываемые управляющей подсистемой управляющие воздействия всегда направлены на уменьшение разнообразия, возможных состояний управляемого объекта, подсистемы. Идеальный вариант характеризуется сведением всех возможных состояний к одному, заранее намеченному. Для этого сознательно создаются необходимые условия и интеллектуальные инструменты, новые материалы и технологии.

Анализ результатов исследований и публикаций по проблеме оптимизации работы управляющей системы позволил выявить наличие различных подходов в решении поставленных задач.

Так, как показал У.Р. Эшби, для того, чтобы управляющая система могла эффективно справляться с этой задачей, она должна сама обладать необходимым разнообразием, так как «только разнообразие может уничтожить разнообразие» ${ }^{1}$. Наиболее совершенной формой саморегулирования с использованием обратной связи является самоорганизация (гомеостазис). Обеспечивается так называемая

\footnotetext{
1 Эшби У.Р. Введение в кибернетику. Москва : ИЛ, 1959. С. 120-125.
} 
сверхстабильность, т.е. способность системы возвращаться к состоянию равновесия после воздействия различных случайных возмущений ${ }^{2}$. Самоорганизующаяся система в принципе является многоконтурной. В ее основе лежит некоторая исходная управляющая система, которая обменивается информацией с объектом управления (первый контур). В результате такого обмена и обеспечения необходимой реакции на «нормальные» внешние воздействия достигается равновесное состояние системы.

Помимо первого контура, в самоорганизующейся системе должен функционировать по крайней мере еще один контур (второго порядка). Это необходимо, чтобы встроенная в него система управления контролировала и прогнозировала эффект, вызываемый деятельностью первого контура.

В том случае, если результаты контроля и прогноза оцениваются системой второго уровня как неудовлетворительные (при отличных от «нормальных» внешних воздействиях), она вносит изменения в правила функционирования первой системы. В принципе, самоорганизующая система обычно имеет больше двух уровней управления ${ }^{3}$. Гармонизация управляющей и управляемых систем ключ к повышению эффективности функционирования организации в целом.

Оптимизация использования ресурсов путем эффективного управления производственными процессами и формирования эффективных организационных структур. Определение факторов, влияющих на состояние функционирования саморегулирующейся системы, в которую включены элементы обратной связи. Обеспечения условий функционирования факторов сверхстабильности, т.е. способности системы возвращаться к состоянию равновесия после воздействия различных случайных возмущений.

В экономических системах переходу к эффективным формам саморегулирования и самоорганизации существенно способствует согласованность производственных целей разных уровней управления и обеспечение эффективного взаимодействия людей, имеющих подчас различные интересы, в направлении достижения этих целей.

${ }^{2}$ Бир С. Кибернетика в управлении производством. Москва : Физматгиз, 1965. C. 271.

3 Бусленко Н.П. и др. Лекции по теории сложных систем. Москва : «Советское радио», 1973. С. 230-236. 
В строительной системе информация по каналам прямой связи передается в виде команд (управляющих воздействий) руководства, по каналам обратной связи - в виде сведений о постоянно меняющейся обстановке на стройке. Кроме того, поступают сведения о возмущающих воздействиях извне. Таким образом, с наиболее общей точки зрения процесс получения информации - процесс снятия неопределенности в результате того, что из некоторой совокупности возможных в данной конкретной ситуации явлений выделяется явление, фактически имевшее место. Основная задача автоматизированной системы управления, сформулированная В.М. Глушковым, состоит в том, чтобы обеспечить оптимальное функционирование объекта управления (производственной организации, объединения, отрасли и т.п.) как единого целого за счет правильного выбора целей и путей их достижения, наилучшего распределения заданий между отдельными частями, из которых состоит объект, и обеспечения их четкого взаимодействия.

\section{1. Задачи, виды и процессы управления}

Известны четыре этапа, характерные для любого процесса управления: появление цели; оценка ситуации; принятие решения и исполнение принятого решения. При управлении сложными процессами оценка ситуации производится на основе собранной и соответствующим образом обработанной информации, выделяются четыре этапа процесса управления:

1) сбор и обработка информации с целью оценки сложившейся ситуации;

2) принятие решения о наиболее целесообразных действиях;

3) исполнение принятого решения;

4) контроль исполнения решения;

Различные виды задач управления отличаются друг от друга способом и последовательностью выполнения этих операций.

Имеется много задач, в которых механизмы сбора информации и исполнения принятого решения отработаны настолько четко, что при осуществлении процесса управления не возникает никаких проблем. В таких задачах все рассмотрение процесса управления сводится, по существу, к рассмотрению только второго этапа. Такие и подобные задачи получили название одноэтапных, или одношаговых задач принятия решения. 
Однако типичным для задач управления является случай. Когда имеющаяся информация бывает или недостаточна для точной оценки ситуации, или искажена посторонними факторами.

\section{1. Управление и исследование операций}

Существует еще один специфический класс задач управления, которые связаны с деятельностью крупных промышленных предприятий или больших строек и могут быть названы задачами организационно-управленческого характера. Каждое отдельное специализированное подразделение крупной организации выполняет определенную часть общей работы, руководствуясь общими целями предприятия или организации. Однако у каждого специализированного подразделения возникают и свои собственные цели. Все эти цели не всегда согласуются, а иногда приходят в противоречие друг с другом. В результате возникает задача организационно-управленческого типа - выработки такой стратегии в отношении запасов, которая была бы наиболее благоприятна для всего предприятия в целом.

Специфическая особенность задач организационноуправленческого типа состоит в том, что последствия того или иного способа их решения могут существенно отразиться на работе всего предприятия. Поэтому принятие окончательного решения всегда относится к компетенции ответственного лица, администратора, наделенного соответствующими правами, и выходит за рамки исследования операций. Исследование операций преследует лишь цель дать в руки администратору обоснованные рекомендации по принятию решения.

\section{2. Управление предприятием в условиях неопределенности. Неопределенность ее виды}

Деятельность хозяйственных субъектов осуществляется в условиях рынка, который характеризуется прежде всего экономической свободой действий производителя. За экономическую свободу необходимо платить, ведь свободе одного предприятия сопутствует свобода и других предприятий, которые могут покупать или не покупать его продукцию, предлагать за нее свои цены, продавать встречную продукцию по определенным ценам, диктовать свои условия сделок, естественно, при этом партнеры стремятся прежде всего к собственной выгоде. Относительность такого понятия, как выгода, сводится к тому, что 
выгода для одних может обернуться убытком для других. Это положение усиливается еще и тем, что предприятия, производящие однородную продукцию, вообще стремятся вытеснить своего конкурента с рынка.

Следовательно, независимо от желания, начиная свою деятельность, а значит, вступая в рынок, любому предприятию придется иметь дело с неопределенностью и, как следствие этого, испытывать на себе на протяжении всей деятельности влияние тех видов рисков, которые присущи данной экономике и данному направлению деятельности.

Специфической характеристикой неопределенности является то, что субъекты предпринимательской деятельности должны учитывать влияние неопределенности развития не только собственного (внутреннего) рынка, но и внешнего, что является следствием расширения мировых хозяйственных связей и процесса интеграции экономик различных стран.

Условия неопределенности, имеющие место в предпринимательской деятельности, являются предметом исследования и объектом постоянного наблюдения экономистов самых различных профилей, а также специалистов других областей (юристов, социологов, политологов и т.п.). Такой комплексный подход к изучению данного явления (неопределенности в бизнесе) связан с тем, что хозяйственные субъекты в процессе своего функционирования испытывают зависимость от целого ряда условий. Данные условия зависимости можно классифицировать по месту их возникновения на такие виды:

- социально-политические;

- административно-законодательные;

- производственные;

- коммерческие;

- финансовые.

Научные представления о неопределенности, а как следствие этого, и о рисках, формировались поэтапно.

В бывшем Советском Союзе в 20-е годы такое понятие, как риск, было закреплено в некоторых законодательных актах относительно производственной и рационализаторской деятельности. Однако уже в середине 30-х годов оно характеризуется как «буржуазное». Становление и последующее функционирование экономики командно-административного типа, одним из важных условий которой являлась всеобщая плановость, явилось следствием того, 446 
что такое понятие, как предпринимательский риск, полностью прекратил свое существование, как явно враждебное плановой экономике.

Разумеется, это имело смысл. Однако полностью с этим нельзя согласиться по нескольким причинам. В первую очередь, потому что и в условиях командной экономики существовала неопределенность, связанная, по крайней мере, с тем, что существовали экспортноимпортные операции, местные товаропроизводители продавали свою продукцию и покупали необходимые товары на внешнем рынке, соприкасаясь таким образом со всеми видами рисков, которые присущи любому субъекту рыночной экономики.

Во-вторых, кроме внешнеэкономических рисков, всегда был риск невыполнения государственных планов, устанавливаемых в приказном порядке. Характерным для того периода времени было то, что последствия этой неопределенности не отражались напрямую на товаропроизводителях, а она целиком и полностью ложилась на плечи государства. Другими словами, существовала такая ситуация, когда при принятии экономических решений субъектами хозяйственной деятельности последние не несли ответственности за последствия решений, а перекладывали ее на общество в целом. Таким образом, отсутствие заинтересованности в результатах экономических решений являлось причиной отсутствия риска как такового.

Ориентация Украины на построение экономики рыночного типа означает появление самых различных видов неопределенности для всех субъектов ее хозяйственной деятельности.

Существует несколько возможных подходов к классификации неопределенности, из которых наиболее широко применим подход ее характеристики с точки зрения вероятности наступления событий.

Причины возникновения неопределенности можно объединить в несколько основных групп:

- недетерминированность процессов, проходящих в обществе в целом и в экономической жизни в частности. При этом недетерминированность является следствием отсутствия возможности полного их предсказания и прогнозирования;

- отсутствие полной информации при планировании поведения рыночного субъекта, либо ее субъективный анализ;

- влияние субъективных факторов на результаты проводимых анализов (уровень квалификации работников, проводящих анализ; величина временного пространства анализируемого периода и т.п.). 


\section{3. Особенности управления фирмой \\ в условиях неопределенности}

В исследованиях по проблемам, связанным с изучением рисков, возникло целое направление работ под общим названием «рискменеджмент», либо, как его еще называют, «управление риском». Его особенностью является комплексность проведения исследований, которая включает в себя различные аспекты: научные, технические, организационно-управленческие, социальноэкономические и т.П. При этом широко применяются методы системного подхода и оптимизации процесса принятия решений в условиях неопределенности с целью разработки методологии управления риском.

В условиях конкурентной борьбы, связанной с постоянно проходящими на рынке изменениями, вопросы управления предприятием претерпевают некоторые изменения, которые проходят по следующим основным направлениям:

- появляется потребность в наличии большого количества аналитического материала, необходимого для анализа и принятия управленческих и маркетинговых решений;

- мониторинговые исследования рынка, сопоставление существующей маркетинговой стратегии и стратегии менеджмента с потребностями рынка;

- появляется необходимость более широкого использования сценарийных подходов при планировании деятельности предприятия (пессимистических, оптимистических и других видов прогнозов с проведением анализа вероятности выпадения событий) и максимально возможного учета всех видов неопределенности.

Факторы неопределенности, которые влияют на любой рыночный субъект, также присутствуют и непосредственно в процессе управления им.

По месту возникновения неопределенность в управлении предприятием (фирмой) может быть следствием:

- неопределенности в определении планового периода и, в частности, периода, на который разрабатывается стратегия развития предприятия;

- неопределенности при формировании целей предприятия и в выборе приоритетов в поставленных целях, что может быть обусловлено наличием ряда альтернативных целей;

- ошибок в оценках настоящего положения дел внутри самого предприятия и его места на рынке, которые, в свою очередь, могут 448 
быть следствием целого ряда причин объективного и субъективного характера;

- недостаточно полной или ошибочной информации о перспективах развития данного предприятия и рынка в целом, а также решений, принятых на ее основании;

- возможных сбоев при разработке стратегии развития предприятия, а также во время ее реализации;

- неопределенности при контроле и оценке результатов деятельности предприятия.

Стратегия развития фирмы в условиях рыночной экономики должна формироваться с учетом этих видов неопределенности на каждом из этапов: на этапе определения периода разрабатываемой стратегии, на этапе формирования целей, при разработке путей реализации выбранной стратегии и формировании направлений деятельности, при анализе компетенций собственного предприятия, а также на этапе контроля за выполнением стратегии и оценки ее реализации. Предложенная методика приводится на рис. 1.

Процесс управления фирмой в условиях неопределенности подвержен влиянию внутренних и внешних факторов.

Внутренние факторы - это те факторы, которые обусловлены неопределенностью, имеющей место на предприятии. Внешние факторы, в отличие от внутренних, являются следствием наличия неопределенности за пределами данного предприятия. 


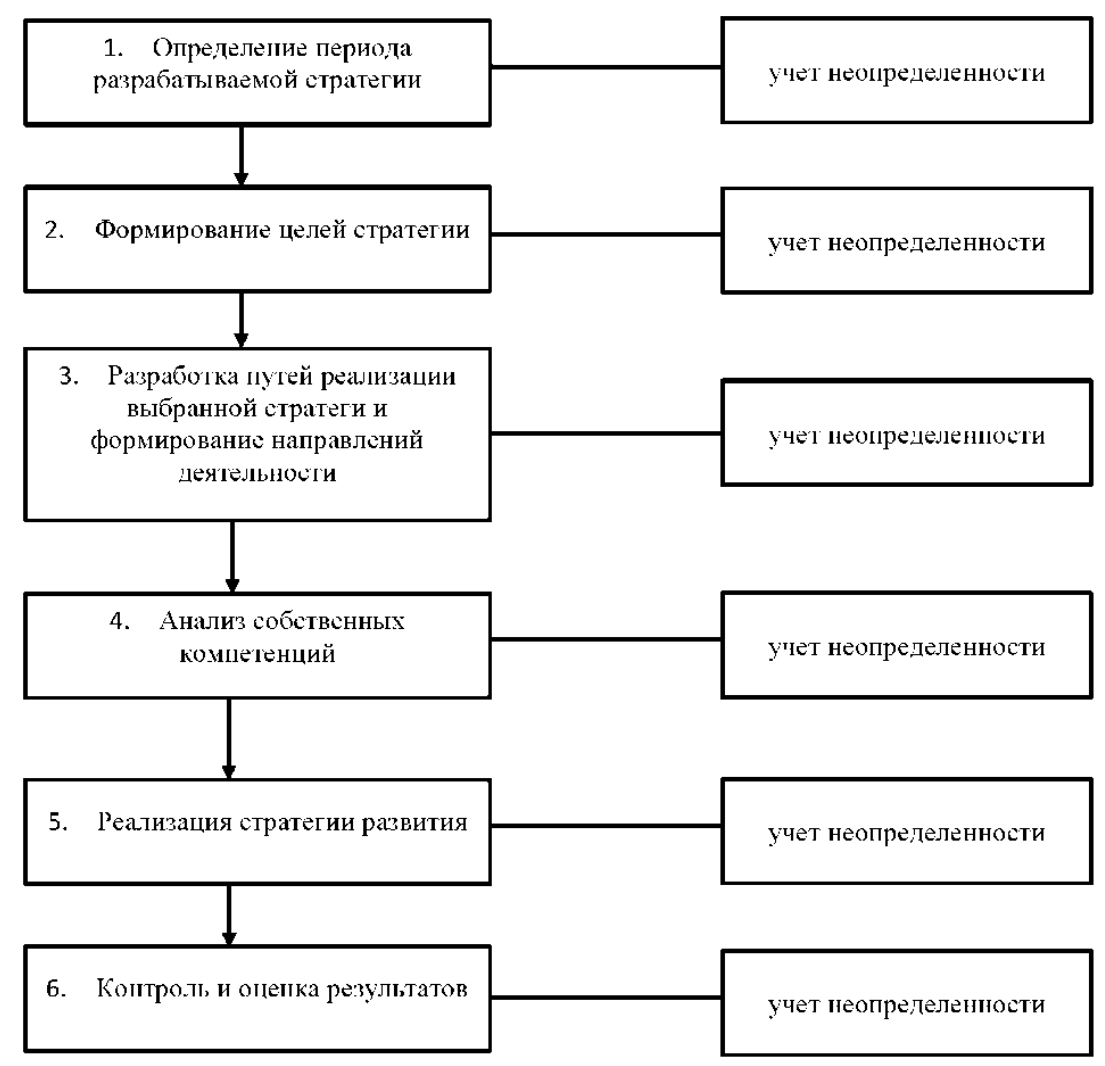

\section{Рис. 1. Стратегическое управление фирмой в условиях неопределенности}

Неопределенность внешнего происхождения является функцией от достоверности внешней информации и, как следствие этого, функцией от достоверности составляемых прогнозов. Чем выше степень неопределенности, тем сложнее принимать управленческое решение.

Некоторые ученые предлагают характеризовать внешнюю среду с точки зрения нестабильности по таким признакам:

- степень обыденности действия (события). По этому признаку события делятся на обычные, т.е. такие, которые случаются довольно часто; неожиданные, но такие, которые имели место в 450 
прошлом; абсолютно неожиданные, и новые, т.е. такие, которые практически не имели аналогов в прошлые периоды;

- темп изменения действия. Под этой характеристикой понимается зависимость между скоростью протекания события и реакцией предприятия (фирмы) на это действие. По этому признаку события классифицируются на: такие, которые протекают медленнее, чем предприятие реагирует на них; такие, скорость протекания которых совпадает со скоростью реакции предприятия; такие, скорость протекания которых превышает скорость реакции на них со стороны предприятия.

Данная характеристика является достаточно целесообразной. Однако вместе с тем она может быть усовершенствована введением характеристики степени прогнозированности дальнейших событий, происходящих во внешней среде, по предложенному ранее механизму оценки степени прогнозированности событий с более детальной разработкой частично прогнозируемых действий.

Наличие источников внутреннего происхождения неопределенности обусловлено тем, что предприятие, являясь сложной, динамичной и открытой системой, имеет в своем составе неоднородные элементы с разнохарактерными связями и особенностями в иерархической структуре.

Динамичность экономической системы является следствием того, что она непрерывно развивается как самостоятельно, так и в результате развития внешнего окружения.

Открытость экономической системы является следствием того, что она существует только лишь как один из элементов более сложной системы (экономики региона, отрасли, страны в целом).

Влияние субъекта управления на управляемый им объект может происходить только лишь при условии обмена информацией между ними. Таким образом, неопределенность в управлении предприятием, с точки зрения качества информации, может возникнуть на этапе: 1) сбора и анализа информации; 2) принятия управленческого решения на основании собранной и проанализированной информации.

Важной функцией управления является планирование. Планирование, как и весь процесс управления, имеет также вероятностный характер. На практике это означает, что неопределенность, существующая при составлении тактических планов, еще более возрастает при составлении стратегических. Тактическое планирование, направленное на определение средств 
для достижения поставленных в стратегическом плане целей, будет иметь тем меньшую неопределенность, чем меньший плановый период оно охватывает и, соответственно, большую неопределенность, чем длиннее плановая перспектива. Таким образом, существует прямо пропорциональная зависимость между сроком, на который разрабатывается план, и степенью его неопределенности. Стремление фирмы охватить своими планами как можно более длительный временной период вступает в явное противоречие, с точки зрения теории рисков, со степенью достоверности планов и возможностью их реализации. Данное противоречие может быть разрешено путем разумной минимизации плановой перспективы и использования вероятностных подходов к оценке конкретных экономических показателей. Никакой план не является окончательным, он может быть пересмотрен при сложившихся условиях. Другими словами, реализованная неопределенность может и должна вызвать в нем адекватные изменения.

\section{2. Оптимизация процесса управления}

\section{1. Критерий качества управления}

Задачу управления рассматривают как математическую задачу. Однако, в отличие от многих других математических задач, она имеет ту особенность, что допускает не одно решение, а множество различных решений. Это связано с тем, что в задачах управления имеется, как правило, много способов организации какого-либо процесса, которые приводят к достижению поставленной цели. Задачу управления можно было бы ставить как задачу нахождения хотя бы одного из возможных способов достижения поставленной цели. Однако такая постановка вопроса обычно бывает недостаточной.

Если имеется множество решений какой-либо задачи, то возникает добавочная задача - выбрать из этого множества решений такое, которое с какой-либо точки зрения является наилучшим.

Математическое выражение, дающее количественную оценку степени выполнения наложенных на способ управления требований, называется критерием качества управления. Наиболее предпочтительным или оптимальным способом управления будет такой, при котором критерий качества управления достигает необходимого значения. 


\section{2. Ограничения, накладываемые на процесс управления}

Задачу нахождения оптимального управления или управления вообще следует считать несуществующей, т.е. не вызывающей никаких проблем, если на характер движения системы не наложено никаких ограничений.

Графическая модель внутренней и внешней среды производственной системы (к примеру, строительной организации) и их взаимодействия в общем виде представлена на рис. 2. Показано также взаимное влияние элементов системы и цели, стоящей перед организацией.

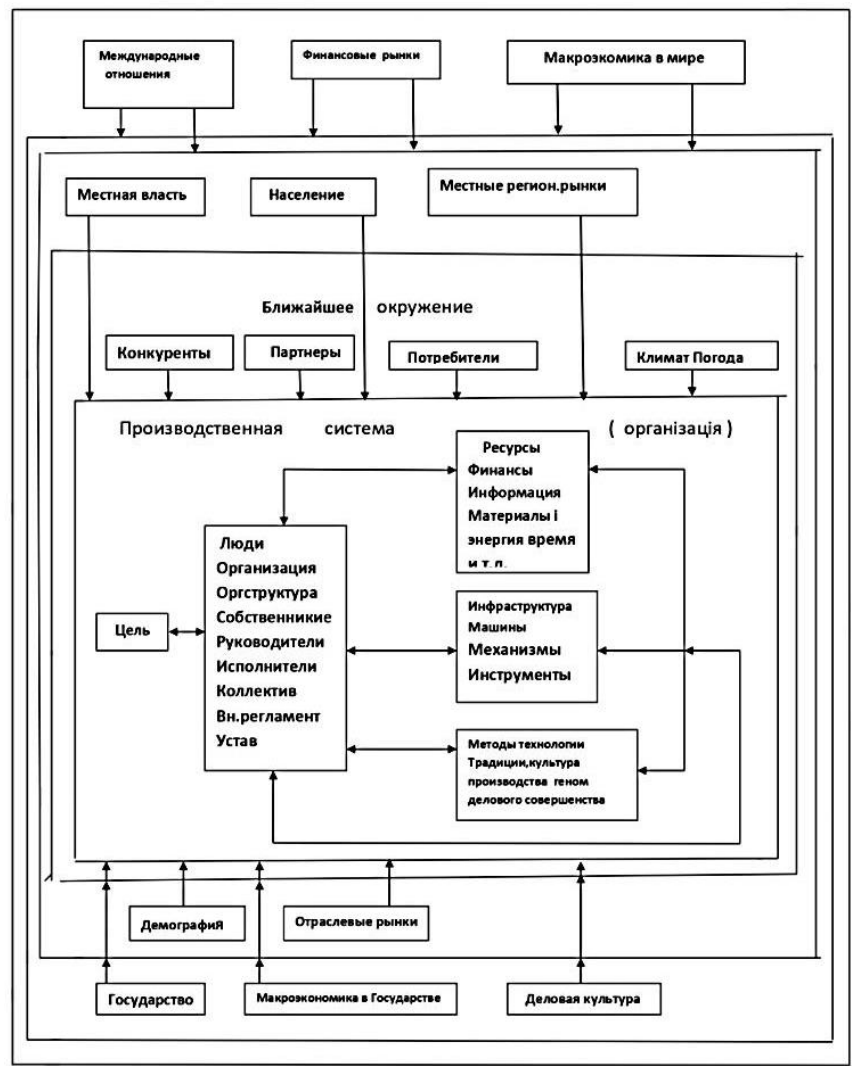

Рис. 2. Внутренняя и внешняя среда организации, связи и взаимодействия 


\section{3. Постановка задачи оптимального управления}

Задачу управления можно считать сформулированной математически, если: сформулирована цель управления, выраженная через критерий качества управления; определены ограничения первого вида, представляющие собой систему дифференциальных или разностных уравнений, ограничивающих возможные способы движения системы; определены ограничения второго вида, представляющие собой систему алгебраических уравнений или неравенств, выражающих ограниченность ресурсов или иных величин, используемых при управлении.

Способ управления, который удовлетворяет всем поставленным ограничениям и соответствует всем критериям качества управления, можно назвать оптимальным управлением.

\section{3. Исследование влияния соционических факторов на параметры технологических процессов производства работ}

\section{в экстремальных условиях}

На производительность труда и его качество, кроме объективных факторов (погодные условия, солнечная радиация, применение новой техники и материалов, способов производства работ и т.д.), оказывают влияние и субъективные факторы, присущие персоналу. Производительность труда отдельно взятого исполнителя зависит от его квалификации, физических данных, выносливости, ловкости, добросовестности.

Производительность работы звена (экипажа) будет определяться, кроме вышеперечисленных качеств, еще и организованностью, слаженностью совместных действий в ограниченном пространстве, в условиях повышенной опасности на протяжении всего рабочего времени, т.е. в экстремальных условиях.

Большое значение при этом имеет и мотивация труда, моральная и материальная заинтересованность. Однако в любом случае при работе двух человек в ограниченном пространстве (люльке) на высоте в экстремальных условиях необходимо учитывать их индивидуальные особенности, совместимость типов характеров.

Отличие в типе личности - это есть не что иное, как отличие в обмене информационными (биоэнергетическими) сигналами с другими типами личности или типами информационного метаболизма.

Известно, что психика человека окружающий мир дифференцирует на определенные составные части или аспекты. И каждый тип 454 
личности о каком-то из этих аспектов получает очень дифференцированную информацию, а информацию о других аспектах воспринимает недифференцированную в сжатом виде. Поэтому разные типы личности, побывав в одной и той же ситуации, помнят и рассказывают совсем другое и другими словами.

В основе психологического дискомфорта человека находится нарушение его информационного метаболизма. Этим нарушением является недополучение нужных сигналов, раздражителей от человека с неподходящими типами психики и перегрузка ненужными, утомляющими, раздражающими сигналами от людей, контакт с которыми затруднен из-за своего типа информационного метаболизма, они свои мысли высказывают малопонятным и неприемлемым способом.

Люди с неприемлемым для кого-то типом информационного метаболизма очень травмируют какими-то замечаниями, которые не дают ни информации, ни покоя.

Всего существуют 16 типов информационного метаболизма. Каждый человек принадлежит к одному из 16 типов. С помощью методов соционики идентифицируется тип личности. В эксперименте использовалась методика и тесты, разработанные А. Аугустинавичуте

В совместной работе люди действуют целенаправленно, эти действия более результативны, если они имеют психическую опору в людях с другим психическими устройством. Это один из секретов простой кооперации и достижения синергизма.

В общении людей и их взаимопонимании важнейшими моментами являются уровень интеллекта и культуры. Но когда речь идет о психическом дополнении психологической совместимости, самое главное - тип информационного метаболизма.

Он определяется порядком селекции и обработки психикой человека (биосистемы) получаемой информации. Этот порядок определяет много психических свойств человека: склонности, способности, интересы, формы активности, целенаправленность и мотивация поведения, взаимоотношения с другими людьми, вкусы и Т.Д.

В соционике существуют способы и методики идентификации информационного метаболизма личности, в частности это можно

${ }^{4}$ Аугустинавичюте А. Теория признаков Рейнина. Соционика, ментология и психология личности. 1998. № № 1-6. 
осуществить путем наблюдения с учетом характерных черт и признаков для каждого типа, а также используя специальные тесты.

Основываясь на основных законах соционики, можно составить диады совместимых типов характеров ${ }^{5}$.

Такой подбор партнеров для длительной работы в экстремальных условиях и формирование экипажей (звеньев) фасадчиков позволяет избежать конфликтных ситуаций наряду с материальной заинтересованностью, квалификацией, добросовестностью и ответственным отношением к своим обязанностям, повысить производительность труда и качество выполняемых работ.

В проводившемся эксперименте на объектах ДСК-3 с рабочими одинаковой квалификации (4 разряд), подобранными с учетом и без учета психологической совместимости, с одинаковой (повышенной в 2 раза, по сравнению с нормативной) материальной заинтересованностью, при благоприятных погодных условиях путем хронометража и сравнения результатов работы по процессам с нормативными был определен поправочный коэффициент $\mathrm{K}_{\text {инт }}$ коэффициент интенсивности труда, учитывающий результаты работы, работая совместимости членов экипажей.

$$
\mathrm{K}_{\text {инт. }}=\frac{\mathrm{T}_{\text {н. }}}{\mathrm{T}_{\text {эксп }}}
$$

где $\mathrm{T}_{\text {эксп }}$ - продолжительность процесса в эксперименте (час); $\mathrm{T}_{\mathrm{H}}-$ нормативня продолжительность процесса (час).

Таблица 1

Коэффициенты интенсивности труда по процессам

\begin{tabular}{|c|c|c|c|c|}
\hline \multirow{2}{*}{$\begin{array}{c}\text { № } \\
\text { п/п }\end{array}$} & Наименование & \multicolumn{3}{|c|}{ Продолжительность процесса Т(час) } \\
\cline { 3 - 5 } & Эксперимент. & Нормативная & Кинт \\
\hline 1. & $\begin{array}{c}\text { Перестановка } \\
\text { самоподъем. люльки }\end{array}$ & 2,5 & 3,3 & 1,326 \\
\hline 2. & Укладка ПРП & 2 & 6,7 & 3,35 \\
\hline 3. & Заделка стыков раствором & 3 & 8,92 & 2,97 \\
\hline 4. & $\begin{array}{c}\text { Праймирование } \\
\text { поверхности стыка }\end{array}$ & 1 & 3,56 & 3,5 \\
\hline 5. & Нанесение мастики & 2 & 4,74 & 2,372 \\
\hline 6. & Устройство солнцезащиты & 0,83 & 2,52 & 3,04 \\
\hline
\end{tabular}
$94 \mathrm{c}$.

5 А. Аугустинавичуте «Дуальна природа людини», «Соціотех». Киев, 1992. 
Результаты проведенного эксперимента свидетельствуют, что подбор экипажей самоподъемных люлек для работы в условиях с повышенной опасностью с учетом типов информационного метаболизма, личности исполнителей влияют на производительность труда и качество работ, поэтому должны учитываться в технологических расчетах в виде поправочного коэффициента $\mathrm{K}_{\text {инт, }}$ определяемого эмпирическим путем.

Влияние соционических факторов на формирование эффективных оргструктур, их управляемость и производительную работу в разных отраслях со сложными условиями деятельности (геологи, военные, строители, линейные работники, горняки и т.д.) доказано также в натурных исследованиях группой киевских ученых-социоников, проведших большую и очень ценную для науки и практики работу в экстремальных условиях Крайнего Севера ${ }^{6}$.

\section{ВЫВОДЫ}

1. Рациональное потребление и расходование всех видов ресурсов (в том числе трудовых, финансовых, времени) возможно лишь при условии четкого и максимально точного планирования, организации и управления производством с учетом внутренней и внешней среды организации, а также методов управления.

2. Исходные данные для проектирования и организации производственных процессов должны максимально соответствовать реальным условиям, быть достоверными.

3. Управление должно быть гибким и устойчивым даже в условиях возможных неопределенностей. Для этого должны максимально использоваться современные компьютерные средства и информационные технологии во главе с высокоинтеллектуальным и компетентным высокоморальным человеком-руководителем.

4. Поскольку имеет место влияние соционических факторов на формирование эффективных оргструктур, их управляемость и производительную работу, необходимо учитывать тип информационного метаболизма личности при формировании социальных производственных структур. Особенно для работы в экстремальных условиях и условиях неопределенности, когда эти факторы приобретают решающее значение.

6 Букалов А.В, Карпенко О.Б, Чикирисова Г.В. О распределении соционических типов в различных производственных коллективах. Соционика,ментология и психология личности. 2000. № 1. 


\section{АННОТАЦИЯ}

В статье исследованы проблематичные аспекты нахождения оптимального управления. В общем случае имеется два вида ограничений, наложенных на выбор способа управления. Ограничениями первого вида являются сами законы природы, в соответствии с которыми происходит функционирование управляемой системы. Графическая модель внутренней и внешней среды производственной системы (к примеру, строительной организации) и их взаимодействие в общем виде представлены в статье. Показано также взаимное влияние элементов системы и цели, стоящей перед организацией. Решающая роль принадлежит персоналу, принимающему и осуществляющему решения по преодолению возникающих перед организацией проблем. Несмотря на преимущества компьютерных систем в быстродействии и работе с большими массивами информации, человек, одаренный интеллектом и интуицией, лучше справляется с работой в условиях неопределенности, тем более с помощью машин. При этом важно учитывать соционические факторы для формирования эффективных организационных структур.

\section{ЛИТЕРАТУРА}

1. Савенко B.І., Пальчик С.П., Клюєва В.В. Победа С.С. Ентропія як прояв системної та діалектичної сутності будівельної організації комбінатного типу. Вип. № 36. КНУБА. Київ, 2018. С. 142-147. URL : http://urss.knuba.edu.UaCopernicusGoogle.

2. Доценко C.I., Савенко В.I. Теоретичне обгрунтування ізоморфізму організаційної структури підприємства. Наук.техн.журнал Енергетика та комл Інтегровані технолог АПК. № 1(6) Харків. 2017. С. 43-47.

3. Савенко В.I. Генетичний підхід до ділової досконалості та ізоморфізм структури будівельної організації. Тексти тез доповідей В.I. Савенко, С.І. Доценко, В.В. Клюєва, С.П. Пальчик. 8 Міжнародна конф.тези КЗЯТПС ЧНТУ. Чернігів, 2018. С. 101-102.

4. Савенко В.І. Оптимальні методи управління будівельною організацією комбінатного типу Текст / В.I. Савенко, С.I. Доценко, В.В. Клюєва, М.О. Терещук. Управління розвитком складних систем. № 35, наук. вид. КНУБА. Київ, 2018. С. 147-154. URL : http://urss.knuba.edu. UaCopernicusGoogle.

5. Савенко В.І. Організаційні виробничі системи в світлі загальної організаційної науки та сучасного кадрового менеджменту. УРСС. 2018. № 34. С. 161-169. URL : http://urss.knuba.edu.UaCopernicusGoogle. 
6. Савенко В.І. Інтелектуальні інформаційні інструменти розвитку виробничої системи енергетичного менеджменту та підприємства в цілому. УРСС. Вun. № 37 КНУБА. Київ, 2019. C. 195-204. URL : http://urss.knuba.edu. UaCopernicusGoogle.

7. Аугустинавичуте А. Дуальна природа людини. Київ, 1992. 95 с.

8. Бухалов О.В., Ермак В.Д., Качанець І.В. Заочная школа соционики. Книга 1. / Соціотех. Украинский институт соционики. К.1001. 95 c.

9. Аугустинавичюте А. Теория признаков Рейнина. Социионика, ментология и психология личности. 1998. № 1-6.

10. Букалов А.В., Карпенко О.Б., Чикирисова Г.В. О распределении соционических типов в различных производственных коллективах. Соционика, ментология и психология личности. 2000. № 1.

11. Карпенко О.Б., Букалов А.В., Чикирисова Г.В. Признаки Рейнина: гендерные различия и социальные ожидания. Соционика, ментология и психология личности. 2000. № 5.

12. Рейнин Г.Р. Теоретический анализ типологических описаний личности в психологии. Сочионика, ментология и психология личности. 1999. № 4-6.

13. Анохин П.К. Принципиальные вопросы общей теории функциональных систем. Москва : Медицина, 1975. 448 с.

14. Велихов, Е.П. Интеллектуальные процессы и их моделирование. Москва : Наука, 1987. 396 с.

15. Доценко С.И. К вопросу о кризисе системной методологии и пути его преодоления. Технологический аудит $u$ резервы производства. 2014.№ 4/1(18). С. 12-17. doi: 10.15587/23128372.2014.26230.

\section{Information about authors:} Savenko V. I.,

Candidate of Technical Sciences (PhD), Doctor of Technical Sciences (RF), Doctor of Civil Engineering, Professor, Kyiv National University of Construction and Architecture 31, Povitroflotskyi ave., Kyiv, 03037, Ukraine

Polosenko A. V., Graduate Student Kyiv National University of Construction and Architecture 31, Povitroflotskyi ave., Kyiv, 03037, Ukraine 